$03,05,11$

\title{
Механизм переноса заряда в новом магнитном топологическом изоляторе $\mathrm{MnBi}_{0.5} \mathrm{Sb}_{1.5} \mathrm{Te}_{4}$
}

\author{
() Н.А. Абдуллаев 1,2 , Х.В. Алигулиева ${ }^{1,3}$, В.Н. Зверев ${ }^{4}$, 3.С. Алиев ${ }^{1,5}$, И.Р. Амирасланов ${ }^{1,2}$, \\ М.Б. Бабанлы ${ }^{2,6}$, З.А. Джахангирли ${ }^{1,2}$, Е.Н. Алиева ${ }^{1}$, Х.Н. Ахмедова ${ }^{1,5}$, Т.Г. Мамедов ${ }^{1}$, \\ М.М. Отроков ${ }^{7,8}$, А.М. Шикин ${ }^{9}$, H.T. Мамедов $^{1}$, Е.В. Чулков ${ }^{9,10}$ \\ ${ }^{1}$ Институт фоизики НАН Азербайджана, \\ Баку, Азербайджан \\ ${ }^{2}$ Бакинский государственный университет, \\ Баку, Азербайджан \\ ${ }^{3}$ Сумгаитский государственный университет, \\ Сумгаит, Азербайджан \\ ${ }^{4}$ Институт фризики твердого тела РАН, \\ Черноголовка, Россия \\ ${ }^{5}$ Азербайджанский государственный университет нефти и промышленности, \\ Баку, Азербайджан \\ ${ }^{6}$ Институт катализа и неорганической химии НАН Азербайджана, \\ Баку, Азербайджан \\ ${ }^{7}$ Centro de Fisica de Materiales (CFM-MPC), Centro Mixto CSIC-UPV/EHU, \\ Donostia-San Sebastian, Basque Country, Spain \\ ${ }^{8}$ IKERBASQUE, Basque Foundation for Science, \\ Bilbao, Spain \\ ${ }^{9}$ Санкт-Петербургский государственный университет, \\ Санкт-Петербург, Россия \\ ${ }^{10}$ Dpto. de Polímeros y Materiales Avanzados: Física, Química y Tecnología, Facultad de Ciencias Químicas, \\ Aptdo. 1072, Donostia-San Sebastián, Basque Country, Spain \\ E-mail: abnadir@mail.ru
}

Поступила в Редакцию 13 апреля 2021 г.

В окончательной редакции 13 апреля 2021 г.

Принята к публикации 18 апреля 2021 г.

\begin{abstract}
Получен новый слоистый магнитный топологический изолятор состава $\mathrm{MnBi}_{0.5} \mathrm{Sb}_{1.5} \mathrm{Te}_{4}$. Исследована электропроводность в плоскости слоев и в направлении перпендикулярном слоям в области температур $1.4-300 \mathrm{~K}$. Обнаружено, что в обоих направлениях в интервале температур 50-300 К наблюдается „металлический“ характер температурной зависимости удельного сопротивления $\rho(T)$. Ниже температуры $T=50 \mathrm{~K}$ величина $\rho$ возрастает и демонстрирует нетривиальную температурную зависимость с особенностью в области критической температуры $T_{c}=23 \mathrm{~K}$. Возрастание сопротивления в температурном интервале $50-23 \mathrm{~K}$ обусловлено спиновыми флуктуациями и магнитным фазовым переходом. Ниже $T_{c}$ и вплоть до $1.4 \mathrm{~K} \rho(T)$ демонстрирует поведение характерное для эффекта слабой локализации, что подтверждается анализом данных, полученных при исследовании магнитосопротивления.
\end{abstract}

Ключевые слова: топологические изоляторы, удельное сопротивление, фазовые переходы, слабая локализация, магнитосопротивление, слоистые кристаллы.

DOI: 10.21883/FTT.2021.08.51154.085

\section{1. Введение}

В последнее время наблюдается новый всплеск интереса к топологическим изоляторам в связи с открытием магнитных топологических изоляторов [1-5]. Топологические изоляторы впервые были теоретически предсказаны еще в 1980-х годах [6]. К настоящему времени экспериментально обнаружены целые классы топологических изоляторов, включая трехмерные (3D) топологические фазы различных соединений [7-17].

Как известно, объем материала с топологической фазой является изолятором, а поверхность - метал- лом. Проводящие свойства поверхности являются следствием сильного спин-орбитального взаимодействия и симметрии относительно обращения времени, которые приводят к возникновению спин-поляризованных топологических поверхностных состояний с дисперсией дираковского типа, т. е. линейной зависимостью энергии от импульса, как в графене. Практический интерес к топологическим изоляторам обусловлен тем, что поверхностные состояния защищены симметрией обращения времени от рассеяния назад на немагнитных примесях и дефектах - и поэтому носители заряда в этих состояниях могут двигаться вдоль поверхности объемного 
материала без потери энергии. Таким образом, возможно осуществление бездиссипативного переноса заряда.

В отличие от немагнитных аналогов, магнитные топологические изоляторы могут иметь щель в конусе Дирака, что делает возможным наблюдение ряда уникальных физических явлений, таких как квантованный аномальный эффект Холла (QAH), магнитоэлектрический эффект, аксионная электродинамика, майорановские фермионы и др. [1,18-21]. Магнитные топологические изоляторы востребованы и в новой области современной электроники - спинтронике, в основном в средствах переноса, записи и хранения информации.

Первые попытки открытия щели в топологическом поверхностном состоянии реализовывались легированием немагнитных топологических изоляторов, например, типа $\mathrm{Bi}_{2} \mathrm{Te}_{3}$ магнитными примесями $\mathrm{Mn}, \mathrm{Cr}, \mathrm{Fe}$ и др. [22-24]. Однако принципиальной трудностью легирования является невозможность равномерного распределения легирующих примесей по объему. Это приводит к тому, что транспортные явления будут определяться наименьшей из всех локальных щелей с существенным понижением температуры реализации вышеперечисленных физических явлений. Другим возможным способом добиться формирования щели в конусе Дирака является формирование ультратонкой пленки двумерного ферромагнетика с магнитным моментом, направленным перпендикулярно пленке, на поверхности немагнитного трехмерного топологического изолятора. Некоторые из теоретически предсказанных систем [25-28] были недавно реализованы экспериментально методом молекулярно-лучевой эпитаксии $[26,28]$. Еще одним классом материалов, в которых происходит расщепление дираковского состояния, являются собственно магнитно упорядоченные топологические изоляторы. Недавно был предложен и экспериментально реализован антиферромагнитный топологический изолятор $\mathrm{MnBi}_{2} \mathrm{Te}_{4}$ [2]. Фотоэмиссионные измерения $[2,4]$ и инфракрасная спектроскопия плазменного края свободных носителей [29] показали, что уровень Ферми в этом соединении пересекает зону проводимости, т.е. лежит выше объемной запрещенной зоны.

Отметим, что практическое наблюдение бездиссипативного переноса заряда в топологических изоляторах сталкивается с большими трудностями. Дело в том, что в топологических изоляторах, наряду с поверхностными носителями заряда, в еще большей концентрации имеются в наличии топологически незащищенные объемные носители, которые могут доминировать в суммарном механизме переноса заряда. Для уменьшения вклада объемных носителей заряда, необходимо, чтобы уровень Ферми находился в запрещенной зоне. Такую корректировку (tuning) положения уровня Ферми можно добиться либо опять-таки легированием, либо же использованием твердых растворов. Теоретически, крайние члены ряда твердых растворов $\mathrm{MnBi}_{2-x} \mathrm{Sb}_{x} \mathrm{Te}_{4}-\mathrm{MnBi}_{2} \mathrm{Te}_{4}$ и
$\mathrm{MnSb}_{2} \mathrm{Te}_{4}$ являются антиферромагнитными топологическими изоляторами $[2,30,31]$. Экспериментально, антиферомагнитное топологическое состояние твердо установлено только для $\mathrm{MnBi}_{2} \mathrm{Te}_{4}$ [2]. Ситуация с $\mathrm{MnSb}_{2} \mathrm{Te}_{4}$ пока остается не ясной ввиду сильного перемешивания атомов между подрешетками $\mathrm{Mn}$ и $\mathrm{Sb}$ в реальных образцах этого соединения. В твердых растворах $\mathrm{MnBi}_{2-x} \mathrm{Sb}_{x} \mathrm{Te}_{4}$ с увеличением доли содержания сурьмы $x$ по данным холловских измерений и по положению уровня Ферми, переход проводимости с $n$-типа в $p$-тип происходит при $x>0.6[32,33]$.

В настоящей работе сообщается о получении и исследовании электрических и гальваномагнитных свойств твердого раствора магнитного топологического изолятоpa $\mathrm{MnBi}_{0.5} \mathrm{Sb}_{1.5} \mathrm{Te}_{4}$.

\section{2. Экспериментальная часть}

Технология получения и характеризация магнитных топологических изоляторов семейства $\mathrm{MnBi}_{2} \mathrm{Te}_{4}\left(\mathrm{Bi}_{2} \mathrm{Te}_{3}\right)_{n}$ подробно описана в работе [34]. Слоистое соединение $\mathrm{MnBi}_{0.5} \mathrm{Sb}_{1.5} \mathrm{Te}_{4}$ кристаллизуется в ромбоэдрическую (rhombohedral) структуру (пространственная группа $R-3 m)$ с осью $c$, перпендикулярной плоскости слоев. На рис. 1 приведена рентгеновская дифрактограмма для соединения $\mathrm{MnBi}_{0.5} \mathrm{Sb}_{1.5} \mathrm{Te}_{4}$. Определенный параметр решетки в направлении оси $c$ равен примерно $41 \AA$.

Электрические и гальваномагнитные (эффект Холла и магнитосопротивление) эффекты в слоистых кристаллах $\mathrm{MnBi}_{0.5} \mathrm{Sb}_{1.5} \mathrm{Te}_{4}$ были изучены стандартным четырехзондовым методом по селективной методике на переменном токе частотой $20.5 \mathrm{~Hz}$ с использованием Lock-in Amplifier - SR830. Величина тока не превышала $1 \mathrm{~mA}$. Исследования проводились в широкой области температур $1.4-300 \mathrm{~K}$. Сильные магнитные поля до $80 \mathrm{kOe}$ были получены с помощью сверхпроводящего соленоида. Образец при гальваномагнитных измерениях помещался в центр соленоида.

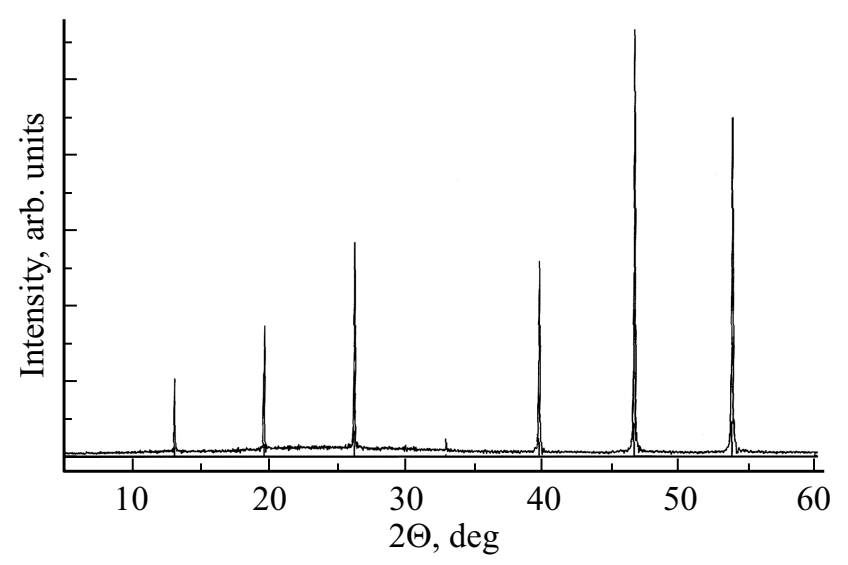

Рис. 1. Рентгеновская дифрактограмма соединения $\mathrm{MnBi}_{0.5} \mathrm{Sb}_{1.5} \mathrm{Te}_{4}$. 
Анизотропия электропроводности в слоистых кристаллах $\mathrm{MnBi}_{0.5} \mathrm{Sb}_{1.5} \mathrm{Te}_{4}$ была изучена усовершенствованным, комбинированным, четырехзондовым методом Montgomery [35], пригодным для образцов, приготовленных в виде тонких пластинок малых размеров. Такие пластинки легко получить из слоистых материалов путем их естественного скола в плоскости, параллельной слоям. Четыре контакта попарно наносятся на противоположные поверхности изучаемого образца в прямоугольной геометрии.

На рис. 2 приведены температурные зависимости удельного сопротивления в плоскости слоев $\left(\rho_{\|}\right)$и в направлении, перпендикулярном слоям $\left(\rho_{\perp}\right)$ соединения $\mathrm{MnBi}_{0.5} \mathrm{Sb}_{1.5} \mathrm{Te}_{4}$, в широкой области температур $1.5-300 \mathrm{~K}$. На вкладке приведена температурная зависимость анизотропии электропроводности $\rho_{\perp} / \rho_{\|}$. Как видно, анизотропия электропроводности практически не меняется с температурой, что свидетельствует об одинаковом механизме переноса заряда в плоскости слоев и в направлении, перпендикулярном слоям.

Для более подробного анализа обратимся к электропроводности в плоскости слоев в другой конфигурации контактов, позволяющей проводить и исследования эффекта Холла. На рис. 3 показана температурная зависимость удельного сопротивления $\rho_{x x}(T)$ соединения $\mathrm{MnBi}_{0.5} \mathrm{Sb}_{1.5} \mathrm{Te}_{4}$ в широкой области температур $1.4-300 \mathrm{~K}$.

Как видно из рис. 3, температурная зависимость удельного сопротивления $\rho_{x x}(T)$ в области температур 50-300 K имеет характерный „металлический“ ход: с уменьшением температуры величина удельного сопротивления уменьшается. Такая температурная зависимость характерна и для классического полупроводника $\mathrm{Bi}_{2} \mathrm{Te}_{3}$ [36]. Вышеизложенное обусловлено тем, что для этих кристаллов при синтезе из расплава стехиометрического состава уже изначально характерно наличие значительного количества собственных точечных дефектов структуры, т.н. антисайтных дефектов и дефектов замещения. Поэтому, выращенные монокристаллы $\mathrm{MnBi}_{0.5} \mathrm{Sb}_{1.5} \mathrm{Te}_{4}$ имеют значительную концентрацию носителей заряда. Согласно исследованиям эффекта Холла, соединение $\mathrm{MnBi}_{0.5} \mathrm{Sb}_{1.5} \mathrm{Te}_{4}$ является полупроводником $p$-типа, с концентрацией дырок в данном образце равной $1.7 \cdot 10^{21} \mathrm{~cm}^{-3}$. Такая большая концентрация примесей формирует в запрещенной зоне значительное количество локальных состояний, образующих широкую примесную зону, перекрывающуюся с собственной зоной чистого кристалла. Поэтому, при исследованиях электропроводности монокристаллов $\mathrm{MnBi}_{0.5} \mathrm{Sb}_{1.5} \mathrm{Te}_{4}$ наблюдается типичный „металлический“ ход температурной зависимости удельного сопротивления.

Далее, ниже температур $T=50 \mathrm{~K}$ величина удельного сопротивления возрастает и зависимость $\rho(T)$ демонстрирует перегиб при критической температуре $T_{c} \sim 23 \mathrm{~K}$ (рис. 3). Этот перегиб обусловлен магнитным фазовым переходом. В соединении $\mathrm{MnBi}_{2} \mathrm{Te}_{4}$ фазовый переход парамагнетик-антиферромагнетик происходит

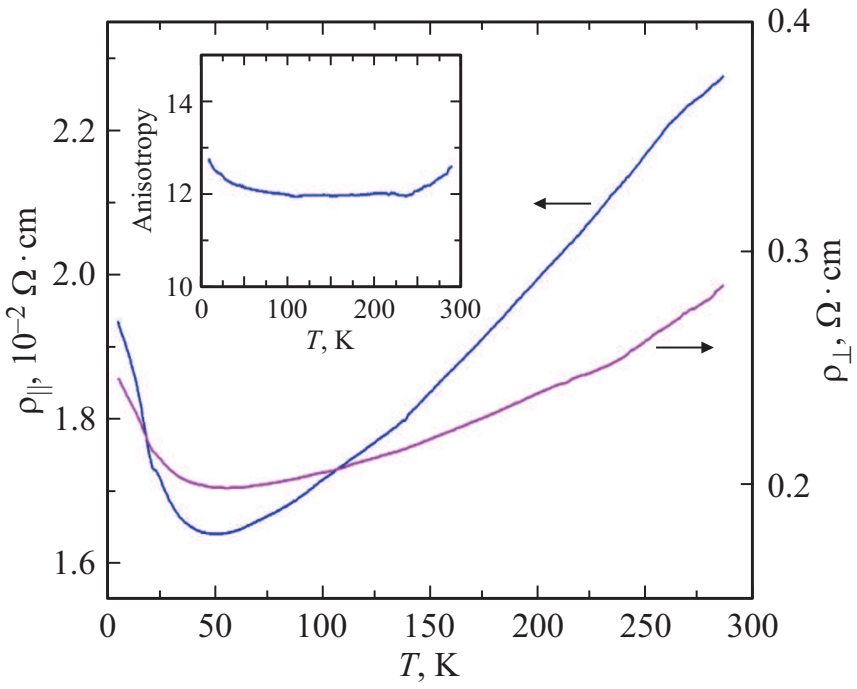

Рис. 2. Температурные зависимости удельного сопротивления соединения $\mathrm{MnBi}_{0.5} \mathrm{Sb}_{1.5} \mathrm{Te}_{4}$ в плоскости слоев $\left(\rho_{\|}\right)$и в направлении, перпендикулярном слоям $\left(\rho_{\perp}\right)$. На вставке: температурная зависимость анизотропии электропроводности $\rho_{\perp} / \rho_{\|}$.

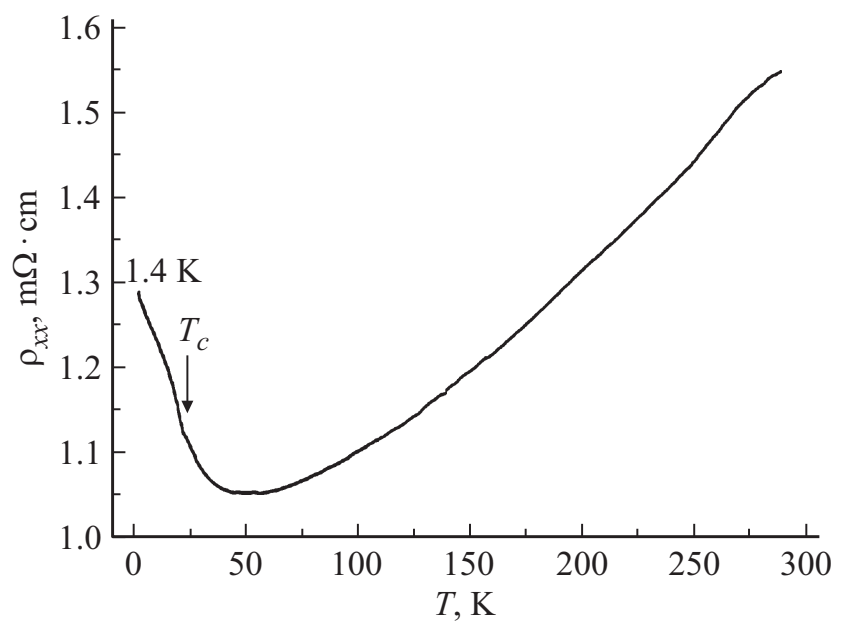

Рис. 3. Температурная зависимость удельного сопротивления $\rho_{x x}(T)$ соединения $\mathrm{MnBi}_{0.5} \mathrm{Sb}_{1.5} \mathrm{Te}_{4}$ в широкой области температур $1.4-300 \mathrm{~K}$.

при $T=25.4 \mathrm{~K}[2]$, а в $\mathrm{MnSb}_{2} \mathrm{Te}_{4}$ магнитный фазовый переход при $T=19 \mathrm{~K}[32]$.

C дальнейшим понижением температуры величина удельного сопротивления продолжает возрастать вследствие локализации носителей заряда при низких температурах, что характерно для „грязных металлов" [37], каковыми, возможно, являются твердые растворы $\mathrm{MnBi}_{0.5} \mathrm{Sb}_{1.5} \mathrm{Te}_{4}$. Локализация носителей заряда (в данном случае дырок) обусловлена интерференцией амплитуд траекторий с самопересечением при рассеянии носителей на примесях и структурных дефектах (т.н. квантовая интерференционная добавка к проводимости, или слабая локализация). В приложенном внешнем маг- 
нитном поле когерентность амплитуд рассеяния нарушается, интерференция амплитуд рассеяния разрушается и сопротивление уменьшается, т.е. наблюдается отрицательное магнитосопротивление (рис. 4).

Следует отметить, что при температуре 5 К уже в слабых магнитных полях напряженностью $(H)$ до $5 \mathrm{kOe}$ сопротивление падает с величины $1.25 \mathrm{~m} \Omega \cdot \mathrm{cm}$ до $1.05 \mathrm{~m} \Omega \cdot \mathrm{cm}$ (рис. 4), что коррелирует с возрастанием сопротивления (рис. 3). Это свидетельствует о том, что в данном случае мы наблюдаем эффект слабой локализации.

Влияние различных по величине внешних магнитных полей $H \leq 10 \mathrm{kOe}$ на температурную зависимость удельного сопротивления $\rho_{x x}(T)$ приведено на рис. 5. Как видно из рис. 5 , с возрастанием величины приложенного магнитного поля (от 0 до $10 \mathrm{kOe}$ ) увеличение сопротивления, вызванное локализацией, постепенно исчеза-

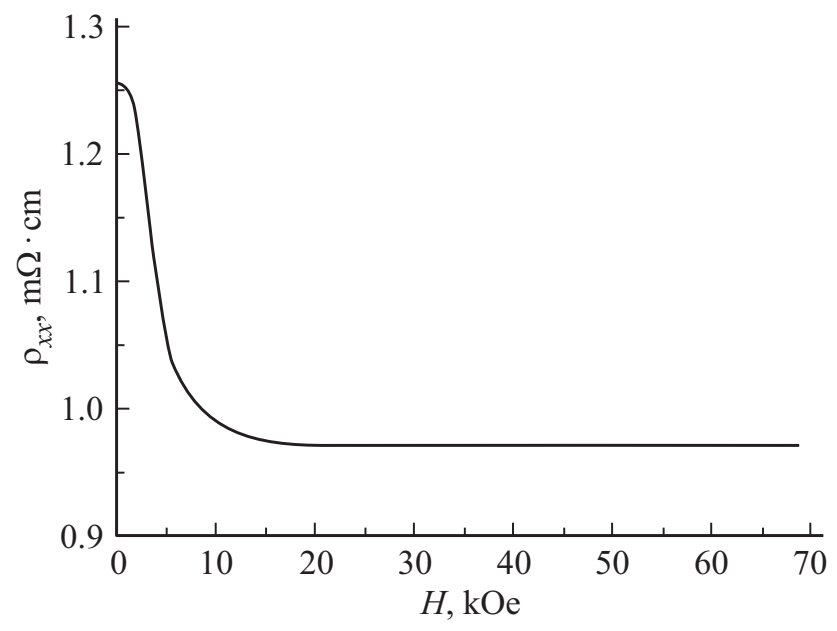

Рис. 4. Зависимость удельного сопротивления $\rho_{x x}$ $\mathrm{MnBi}_{0.5} \mathrm{Sb}_{1.5} \mathrm{Te}_{4} \quad$ от $\quad$ внешнего магнитного поля $H$ при температуре $T=5 \mathrm{~K}$.

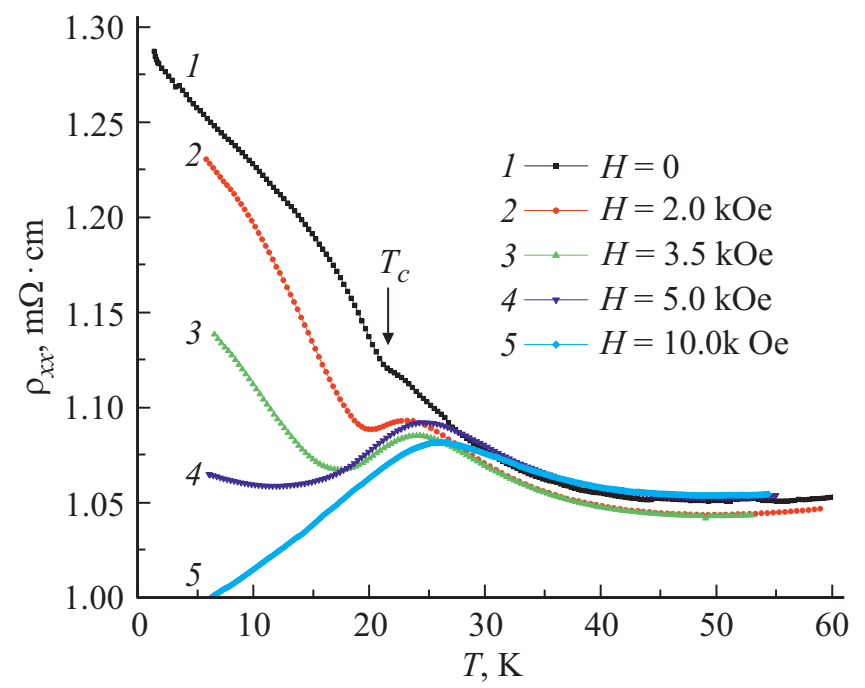

Рис. 5. Температурная зависимость удельного сопротивления $\rho_{x x}$ в $\mathrm{MnBi}_{0.5} \mathrm{Sb}_{1.5} \mathrm{Te}_{4}$ при внешних магнитных полях напряженностью $0 \leq H \leq 10 \mathrm{kOe}$.

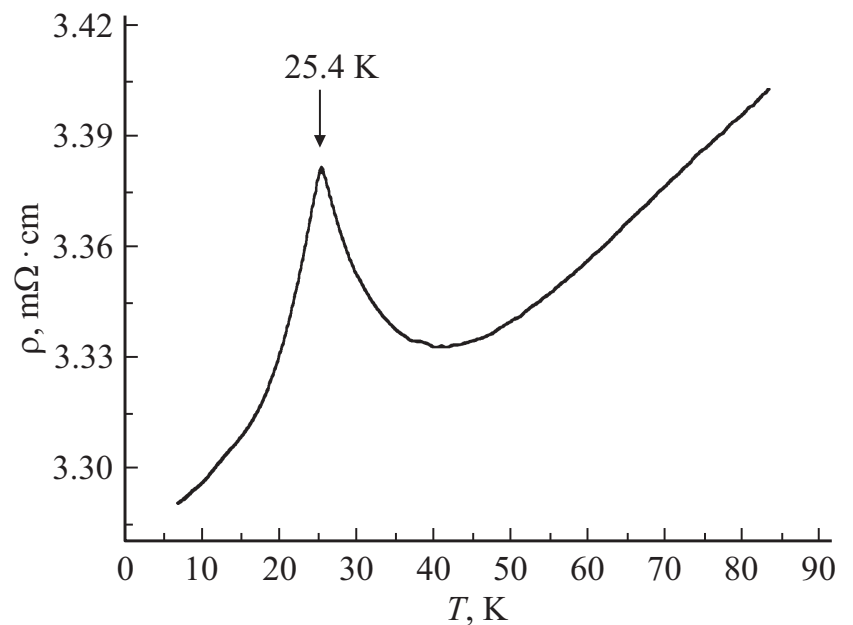

Рис. 6. Температурная зависимость удельного сопротивления $\rho \mathrm{MnBi}_{2} \mathrm{Te}_{4}[2]$.

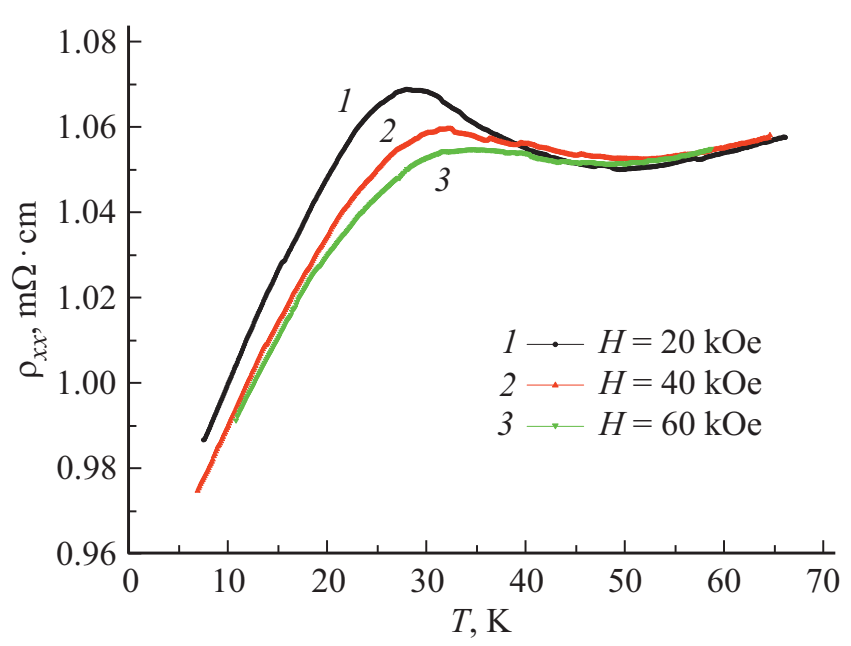

Рис. 7. Температурная зависимость удельного сопротивления $\rho_{x x}$ в $\mathrm{MnBi}_{0.5} \mathrm{Sb}_{1.5} \mathrm{Te}_{4}$ при внешних магнитных полях напряженностью $20 \leq H \leq 60 \mathrm{kOe}$.

ет и отчетливо виден пик на $\rho_{x x}(T)$, обусловленный магнитным фазовым переходом. Аналогичный пик на зависимости $\rho_{x x}(T)$ наблюдался нами и в монокристаллах $\mathrm{MnBi}_{2} \mathrm{Te}_{4}$ [2] (рис. 6), обусловленный магнитным фазовым переходом парамагнетик-антиферромагнетик. Возникновение пика обусловлено рассеянием носителей заряда на спиновых флуктуациях при магнитном фазовом переходе.

Как видно из рис. 5, приложенное поле $H=5 \mathrm{kOe}$ практически ликвидирует возрастание сопротивления, вызванное слабой локализацией, что является дополнительным потверждением наблюдения эффекта слабой локализации.

Сравнение рис. 5 и 6 показывает, что пик на зависимости $\rho_{x x}(T)$ в $\mathrm{MnBi}_{0.5} \mathrm{Sb}_{1.5} \mathrm{Te}_{4}$ более размыт, чем в $\mathrm{MnBi}_{2} \mathrm{Te}_{4}$. Это обусловлено тем, что в твердом растворе $\mathrm{MnBi}_{0.5} \mathrm{Sb}_{1.5} \mathrm{Te}_{4}$ больше дефектов, что приводит к размытию температуры фазового перехода. 
Дальнейшее увеличение величин внешних магнитных полей (от 20 до $60 \mathrm{kOe}$ ) приводит к постепенному уменьшению пика на зависимости $\rho_{x x}(T)$ (рис. 7), что свидетельствует о магнитном происхождении этой особенности. Необходимо однако отметить, что достаточно сильное магнитное поле $H=60 \mathrm{kOe}$ не ликвидирует полностью пик на зависимости $\rho_{x x}(T)$ в твердом раствоpe $\mathrm{MnBi}_{0.5} \mathrm{Sb}_{1.5} \mathrm{Te}_{4}$, что указывает на достаточно сильное обменное взаимодействие.

\section{3. Заключение}

Нами получены и охарактеризованы новые магнитные топологические изоляторы твердого раствора $\mathrm{MnBi}_{0.5} \mathrm{Sb}_{1.5} \mathrm{Te}_{4}$. Из исследований анизотропии электропроводности выявлен одинаковый механизм переноса заряда в плоскости слоев и в направлении, перпендикулярном слоям, в широкой области температур 1.4-300 K. Обнаружено, что в интервале температур 50-300 K наблюдается „металлический“ характер температурной зависимости удельного сопротивления. Ниже температур $T=50 \mathrm{~K}$ величина удельного сопротивления возрастает и зависимость $\rho(T)$ демонстрирует перегиб при критической температуре $T_{c}=23 \mathrm{~K}$. Возрастание сопротивления в этом температурном интервале обусловлено спиновыми флуктуациями и происходящим магнитным фазовым переходом. Ниже температуры $23 \mathrm{~K}$ возрастание сопротивления обусловлено эффектом слабой локализации, на что указывают данные исследований влияния внешнего магнитного поля на электропроводность.

\section{Финансирование работы}

Настоящая работа была выполнена при финансовой поддержке Фонда развития науки при Президенте Азербайджанской Республики (гранты № EİF-BGM4-RFTF-1/2017-21/04/1-M-02 и EİF/MQM/Elm-Tehsil-12016-1(26)-71/16/1), Российского фонда фундаментальных исследований (грант № 18-52-06009), гранта № 73028629 Санкт-Петербургского университета, а также фонда Spanish Ministerio de Ciencia e Innovación (Grant N PID2019-103910GB-I00).

\section{Конфликт интересов}

Авторы заявляют, что у них нет конфликта интересов.

\section{Список литературы}

[1] Y. Tokura, K. Yasuda, A. Tsukazaki. Nature Rev. Phys. 1, 126 (2019).

[2] M.M. Otrokov, I.I. Klimovskikh, H. Bentmann, D. Estyunin, A. Zeugner, Z.S. Aliev, S. Gaß, A.U.B. Wolter, A.V. Koroleva, A.M. Shikin, M. Blanco-Rey, M. Hoffmann, I.P. Rusinov, A.Yu. Vyazovskaya, S.V. Eremeev, Yu.M. Koroteev, V.M. Kuznetsov, F. Freyse, J. Sánchez-Barriga, I.R. Amiraslanov, M.B. Babanly, N.T. Mamedov, N.A. Abdullayev, V.N. Zverev,
A. Alfonsov, V. Kataev, B. Büchner, E.F. Schwier, S. Kumar, A. Kimura, L. Petaccia, G. Di Santo, R.C. Vidal, S. Schatz, K. Kißner, M. Ünzelmann, C.H. Min, S. Mose, T.R.F. Peixoto, F. Reinert, A. Ernst, P.M. Echenique, A. Isaeva, E.V. Chulkov. Nature 576, 416 (2019).

[3] H. Li, S.-Y. Gao, S.-F. Duan, Y.-F. Xu, K.-J. Zhu, S.-J. Tian, W.-H. Fan, Z.-C. Rao, J.-R. Huang, J.-J. Li, Z.-T. Liu, W.-L. Liu, Y.-B. Huang, Y.-L. Li, Y. Liu, G.-B. Zhang, H.-C. Lei, Y.-G. Shi, W.-T. Zhang, H.-M. Weng, T. Qian, H. Ding. Phys. Rev. X 9, 041039 (2019).

[4] I.I. Klimovskikh, M.M. Otrokov, D. Estyunin, S.V. Eremeev, S.O. Filnov, A. Koroleva, E. Shevchenko, V. Voroshnin, A.G. Rybkin, I.P. Rusinov, M. Blanco-Rey, M. Hoffmann, Z.S. Aliev, M.B. Babanly, I.R. Amiraslanov, N.A. Abdullayev, V.N. Zverev, A. Kimura, O.E. Tereshchenko, K.A. Kokh, L. Petaccia, G. Di Santol, A. Ernst, P.M. Echenique, N.T. Mamedov, A.M. Shikin, E.V. Chulkov. npj Quantum Materials 5, $54(2020)$.

[5] L. Ding, C. Hu, F. Ye, E. Feng, N. Ni, H. Cao. Phys. Rev. B 101, 020412 (2020).

[6] Б.А. Волков, О.А. Панкратов. Письма в ЖЭТФ 42, 4, 145 (1985).

[7] M. König, S. Wiedmann, C. Brüne, A. Roth, H. Buhmann, L.W. Molenkamp, X.L. Qi, S.C. Zhang. Science 318, 766 (2007).

[8] Y. Xia, D. Qian, D. Hsieh L. Wray, A. Pal, H. Lin, A. Bansil, D. Grauer, Y.S. Hor, R.J. Cava, M.Z. Hasan. Nature Phys. 5, 398 (2009).

[9] H. Zhang, C.X. Liu, X.L. Qi, X. Dai, Z. Fang, S.C. Zhang. Nature Phys. 5, 438 (2009).

[10] K. Kuroda, M. Ye, A. Kimura, S.V. Eremeev, E.E. Krasovskii, E.V. Chulkov, Y. Ueda, K. Miyamoto, T. Okuda, K. Shimada, H. Namatame, M. Taniguchi. Phys. Rev. Lett. 105, 146801 (2010).

[11] Y.L. Chen, Z.K. Liu, J.G. Analytis, J.-H. Chu, H.J. Zhang, B.H. Yan, S.-K. Mo, R.G. Moore, D.H. Lu, I.R. Fisher, S.C. Zhang, Z. Hussain, Z.-X. Shen. Phys. Rev. Lett. 105, 266401 (2010).

[12] H. Lin, R.S. Markiewicz, L.A. Wray, L. Fu, M.Z. Hasan, A. Bansil. Phys. Rev. Lett. 105, 036404 (2010).

[13] S.V. Eremeev, G. Bihlmayer, M. Vergniory, Yu.M. Koroteev, T.V. Menshchikova, J. Henk, A. Ernst, E.V. Chulkov. Phys. Rev. B 83, 205129 (2011).

[14] S. Kim, M. Ye, K. Kuroda, Y. Yamada, E.E. Krasovskii, E.V. Chulkov, K. Miyamoto, M. Nakatake, T. Okuda, Y. Ueda, K. Shimada, H. Namatame, M. Taniguchi, A. Kimura. Phys. Rev. Lett. 107, 056803 (2011).

[15] S.V. Eremeev, G. Landolt, T.M. Menshchikova, B. Slomski, Yu.M. Koroteev, Z.S. Aliyev, M.B. Babanly, J. Henk, A. Ernst, L. Patthey, A. Eich, A.A. Khajetoorians, J. Hagemeister, O. Pietzsch, J. Weibe, R. Wiesendanger, P.M. Echenique, S. Tsirkin, I.R. Amiraslanov, J.H. Dil, E.V. Chulkov. Nature Commun. 3, 635 (2012).

[16] J. Henk, A. Ernst, S.V. Eremeev, E.V. Chulkov, I.V. Maznichenko, I. Mertig. Phys. Rev. Lett. 108, 206801 (2012).

[17] M. Papagno, S.V. Eremeev, J. Fujii, Z.S. Aliev, M.B. Babanly, S.K. Mahatha, I. Vobornik, N.T. Mamedov, D. Pacile, E.V. Chulkov. ACS nano 10, 3, 3518 (2016).

[18] C.-Z. Chang, J. Zhang, X. Feng, J. Shen, Z. Zhang, M. Guo, K. Li, Y. Ou, P. Wei, L.L. Wang, Z.-Q. Ji, Y. Feng, S. Ji, X. Chen, J. Jia, X. Dai, Z. Fang, S.-C. Zhang, K. He, Y. Wang, L. Lu, X.-C. Ma, Q.-K. Xue. Science 340, 167 (2013).

[19] Q.L. He, L. Pan, A.L. Stern, E.C. Burks, X.Che, G. Yin, J. Wang, B. Lian, Q. Zhou, E.S. Choi, K. Murata, X. Kou, Z. Chen, T.Nie, Q. Shao, Y. Fan, S.-C. Zhang, K. Liu, J. Xia, K.L. Wang. Science 357, 294 (2017). 
[20] A.M. Essin, J.E. Moore, D. Vanderbilt. Phys. Rev. Lett. 102, 146805 (2009).

[21] R. Li, J. Wang, X.-L. QiandS.-C. Zhang. Nature Phys. 6, 284 (2010).

[22] J. Choi, H.-W. Lee, B.-S. Kim, S. Choi, J. Choi, J.H. Song, S. Cho. J. Appl. Phys. 97, 10D324 (2005).

[23] J.W.G. Bos, M. Lee, E. Morosan, H.W. Zandbergen, W.L. Lee, N.P. Ong, R.J. Cava. Phys. Rev. B 74, 184429 (2006).

[24] Y.S. Hor, P. Roushan, H. Beidenkopf, J. Seo, D. Qu, J.G. Checkelsky, L.A. Wray, D. Hsieh, Y. Xia, S.-Y. Xu, D. Qian, M.Z. Hasan, N.P. Ong, A. Yazdani, R.J. Cava. Phys. Rev. B 81, 195203 (2010).

[25] M.M. Otrokov, T.V. Menshchikova, M.G. Vergniory, I.P. Rusinov, A.Yu. Vyazovskaya, Yu.M. Koroteev, G. Bihlmayer, A. Ernst, P.M. Echenique, A. Arnau, E.V. Chulkov. 2D-Materials 4, 025082 (2017).

[26] T. Hirahara, S.V. Eremeev, T. Shirasawa, Y. Okuyama, T. Kubo, R. Nakanishi, R. Akiyama, A. Takayama, T. Hajiri, S.-I. Ideta, M. Matsunami, K. Sumida, K. Miyamoto, Y. Takagi, K. Tanaka, T. Okuda, T. Yokoyama, S.-I. Kimura, S. Hasegawa, E.V. Chulkov. Nano Lett. 17, 3493 (2017).

[27] M.M. Otrokov, T.V. Menshchikova, I.P. Rusinov, M.G. Vergniory, V.M. Kuznetsov, E.V. Chulkov. JETP Lett. 105, 297 (2017).

[28] T. Hirahara, M.M. Otrokov, T. Sasaki, K. Sumida, Y. Tomohiro, S. Kusaka, Y. Okuyama, S. Ichinokura, M. Kobayashi, Y. Takeda, K. Amemiya, T. Shirasawa, S. Ideta, K. Miyamoto, K. Tanaka, S. Kuroda, T. Okuda, K. Hono, S.V. Eremeev, E.V. Chulkov. Nature Commun. 11, 4821 (2020).

[29] B. Xu, Y. Zhang, E.H. Alizade, Z.A. Jahangirli, F. Lyzwa, E. Sheveleva, P. Marsik, Y.K. Li, Y.G. Yao, Z.W. Wang, B. Shen, Y.M. Dai, V. Kataev, M.M. Otrokov, E.V. Chulkov, N.T. Mamedov, Ch. Bernhard. Phys. Rev. B 103, L121103 (2021).

[30] R.C. Vidal, H. Bentmann, T.R.F. Peixoto, A. Zeugner, S.K. Moser, C.H. Min, S. Schatz, K. Kißner, M. Unzelmann, C. Fornari, H.B. Vasili, M. Valvidares, K. Sakamoto, J. Fujii, I. Vobornik, T.K. Kim, R. Koch, C. Jozwiak, A. Bostwick, J. Denlinger, E. Rotenberg, M.M. Otrokov, E.V. Chulkov, M. Ruck, A. Isaeva, F. Reinert. Phys. Rev. B 100, 121104R (2019).

[31] S.V. Eremeev, I.P. Rusinov, Yu.M. Koroteev, A.Yu. Vyazovskaya, M. Hoffmann, P.M. Echenique, A. Ernst, M.M. Otrokov, E.V. Chulkov. Препринт на https://arxiv.org/abs/2102.02532 (2021).

[32] J.-Q. Yan, S. Okamoto, M.A. McGuire, A.F. May, R.J. Mc Queeney, B.C. Sales. Phys. Rev. B 100, 104409 (2019).

[33] B. Chen, F. Fei, D. Zhang, B. Zhang, W. Liu, S. Zhang, P. Wang, B. Wei, Y. Zhang, Z. Zuo, J. Guo, Q. Liu, Z. Wang, X. Wu, J. Zong, X. Xie, W. Chen, Z. Sun, S. Wang, Y. Zhang, M. Zhang, X. Wang, F. Song, H. Zhang, D. Shen, B. Wang. Nature Commun. 10, 4469 (2019).

[34] Z.S. Aliev, I.R. Amiraslanov, D.I. Nasonova, A.V. Shevelkov, N.A. Abdullayev, Z.A. Jahangirli, E.N. Orujlu, M.M. Otrokov, N.T. Mamedov, M.B. Babanly, E.V. Chulkov. J. Alloys Comp. 789, 443 (2019).

[35] Л.И. Буравов. ЖТФ 59, 4, 138 (1989).

[36] Н.А. Абдуллаев, С.Ш. Кахраманов, Т.Г. Керимова, К.М. Мустафаева, С.А. Немов. ФТП 43, 2, 156 (2009).

[37] В.Ф. Гантмахер. Электроны в неупорядоченных средах. Физматлит, М. (2013). 288 с.

Редактор Т.Н. Василевская 